Supporting Information for

\title{
Effect of Local Atomic Structure on Sodium Ion Storage in Hard Amorphous Carbon
}

\author{
Jiuhui Han, Isaac Johnson, Zhen Lu, Akira Kudo, Mingwei Chen* \\ *Corresponding author. Email: mwchen@jhu.edu
}

Supporting Information Including:

Experimental Procedures

Figures S1-S12

Tables S1-S2

References 


\section{Experimental Procedures}

Synthesis of the hard amorphous carbon (a-C). The synthetic procedure for a-C has been reported in detail in our previous study ${ }^{1}$, which is also schematically shown here as Figure $\mathrm{S} 1$. Briefly, cold-rolled $\mathrm{Ni}_{30} \mathrm{Mn}_{70}$ (atomic ratio) sheets with a thickness of 50 $\mu \mathrm{m}$ was first dealloyed in a $1.0 \mathrm{M}\left(\mathrm{NH}_{4}\right)_{2} \mathrm{SO}_{4}$ aqueous solution at $50^{\circ} \mathrm{C}$ for $5 \mathrm{~h}$ to produce the nanoporous $\mathrm{Ni} .0 .4 \mathrm{~g}$ of the as-prepared nanoporous $\mathrm{Ni}$ was then added into the mixture solution of $80 \mathrm{~mL}$ triethylene glycol and $260 \mu \mathrm{L} 25 \mathrm{M}$ aqueous $\mathrm{NaOH}$, and then kept in a $230^{\circ} \mathrm{C}$ well-stirred oil bath for 14 hours to prepare mesoporous $\mathrm{Ni}_{3} \mathrm{C}$. The mesoporous $\mathrm{Ni}_{3} \mathrm{C}$ was collected, washed and then used as the precursor for preparing a-C by chemical dealloying. The dealloying was carried out by immersing the mesoporous $\mathrm{Ni}_{3} \mathrm{C}$ sheets in a $0.5 \mathrm{M} \mathrm{FeCl}_{3}$ aqueous solution at room temperature over night. The obtained a-C samples were further etched with $2.0 \mathrm{M} \mathrm{HCl}$ acid solution at $50^{\circ} \mathrm{C}$ for 12 hours to completely remove $\mathrm{Ni}$ and to avoid $\mathrm{Fe}$ adsorption. Finally, the freestanding a-C sheets were thoroughly rinsed with deionized water and then dried by supercritical $\mathrm{CO}_{2}$.

Thermal treatment of a-C. The a-C-900 samples were prepared by annealing the as-prepared a-C sheets in a tube furnace at $900^{\circ} \mathrm{C}$ under Ar protection for 1 hour. The heating rate was $20^{\circ} \mathrm{C} / \mathrm{min}$ and the Ar flow rate was $800 \mathrm{sccm}$. Thermal treatments at higher temperatures of 1300 and $1800^{\circ} \mathrm{C}$ were carried out using a ultrahigh temperature furnace (TCF-500, Thermocera, Japan) with $1000 \mathrm{sccm}$ Ar flowing. The temperature was ramped at a rate of $20^{\circ} \mathrm{C} / \mathrm{min}$ to $1400^{\circ} \mathrm{C}$ and then at a rate of $10^{\circ} \mathrm{C} / \mathrm{min}$ to higher temperatures.

Microstructural and chemical characterization. The microstructure of the samples was investigated using a field-emission scanning electron microscope (JEOL JIB-4600F) equipped with an energy dispersive X-ray spectrometer and a field-emission transmission electron microscope (JEOL JEM-2100F, $200 \mathrm{keV}$ ) with double spherical aberration correctors for both the probe-forming and image-forming objective lenses. X-ray diffraction was carried out using a RIGAKU SmartLab 9MTP 
diffractometer with a $9.0 \mathrm{~kW}$ rotating anode generator $(\mathrm{Cu} \mathrm{K} \alpha 1$ radiation; $\lambda=1.5406$ $\AA$ ) at a scan rate of $0.4^{\circ}$ per min. Raman spectra were recorded using a micro-Raman spectrometer (Renishaw InVia RM 1000) with an incident wavelength of $514.5 \mathrm{~nm}$ at a low laser power of $10.0 \mathrm{~mW}$. Specific surface areas and mesopore size distributions of the samples were measured by $\mathrm{N}_{2}$ adsorption-desorption using BELSORP-mini II (BEL. JAPAN. INC) at 77.0 K. Pore size distribution in the micropore range was measured by $\mathrm{CO}_{2}$ adsorption-desorption at 273K using QUADRASORB EVO4 (Quantachrome Instruments). Samples were dried at $100^{\circ} \mathrm{C}$ under vacuum for 12 hours before the measurements. Chemical compositions of the samples were characterized by an X-ray photoelectron spectroscopy (XPS, AXIS ultra DLD, Shimazu) in a vacuum of $10^{-7} \mathrm{~Pa}$ with an $\mathrm{Al} \mathrm{K} \alpha$ (mono) anode.

Electrochemical characterization. The a-C electrodes were paired with sodium foils and installed into R2032-type coin cells (MTI Corporation) to investigate the electrochemical performances as well as kinetic behaviors in $\mathrm{Na}^{+}$-containing non-aqueous electrolytes. The cells were assembled inside an argon-filled glove box (water and oxygen content $<1 \mathrm{ppm}$ ) using a well-cut free-standing a-C sheet as the working electrode, a fresh sodium foil (Sigma Aldrich) as the counter/reference electrode, and a Whatman ${ }^{\circledR}$ glass fiber membrane as the separator. No binders or conducting carbon were used for the electrodes. $1.0 \mathrm{M} \mathrm{NaPF} 6$ in ethylene carbonate/dimethyl carbonate $(1: 1 \mathrm{v} / \mathrm{v})$ was employed as the electrolyte. Areal mass loading of the electrodes was $\sim 0.82 \mathrm{mg} \mathrm{cm}^{-2}$. Galvanostatic discharge/charge tests were conducted at room temperature using a Hokuto discharging/charging system. The current densities and specific capacities were calculated based on the loading mass of the a-C electrodes. Cyclic voltammetry measurements were conducted in the potential window from 0.01 to $2.5 \mathrm{~V}$ using a VersaSTAT3 electrochemical workstation (Princeton Applied Research, USA). The potentials are reported vs. $\mathrm{Na} / \mathrm{Na}^{+}$. 
Nanoporous Ni

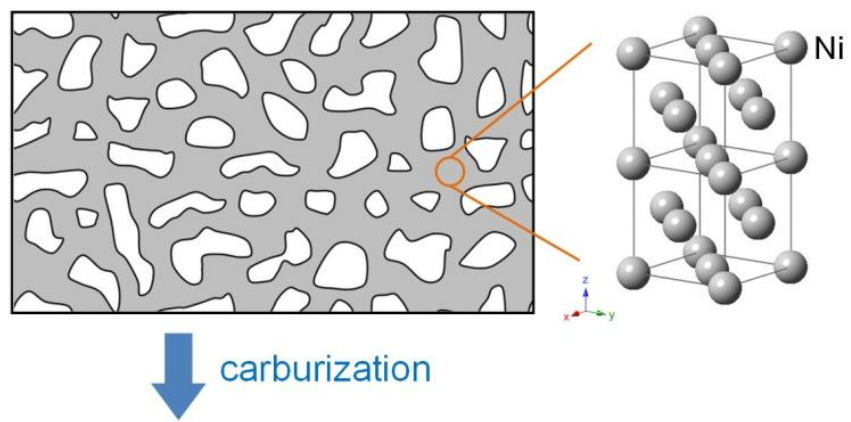

Mesoporous $\mathrm{Ni}_{3} \mathrm{C}$

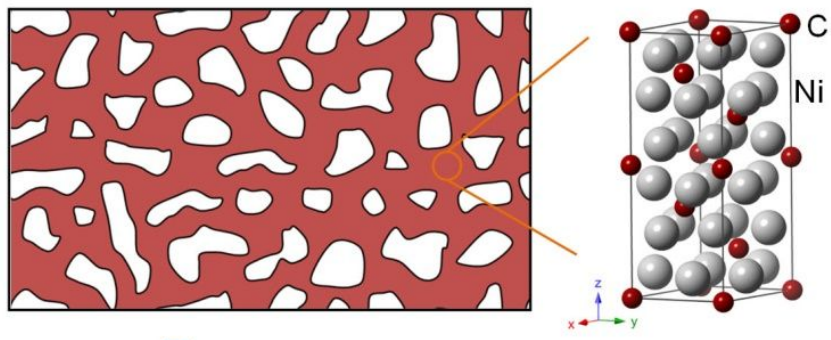

dealloying/etching

Amorphous carbon

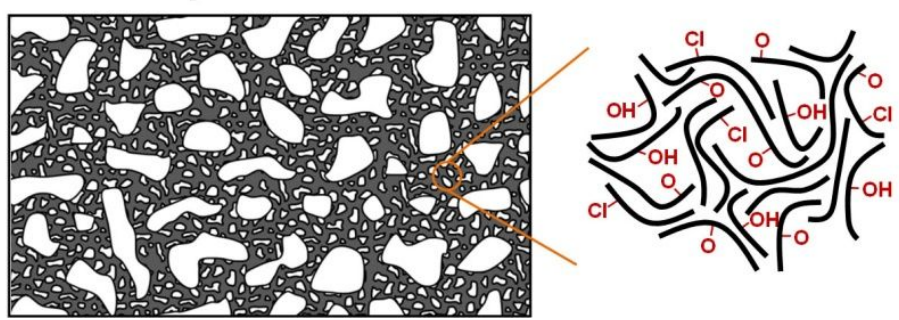

Figure S1. Schematic illustration of the synthetic procedure for a-C. Note that the use of a mesoporous $\mathrm{Ni}_{3} \mathrm{C}$ rather than a bulk $\mathrm{Ni}_{3} \mathrm{C}$ precursor is critical for achieving room-temperature dealloyed hard carbon since the large surface area and short diffusion length enable low-temperature $\mathrm{Ni}$ carbonization and $\mathrm{Ni}$ dissolution in reasonable time. However, the mesopores inheriting from the $\mathrm{Ni}_{3} \mathrm{C}$ precursor have no distinct effect on the mechanistic study of sodium sotrage in amorphous carbon. 
a

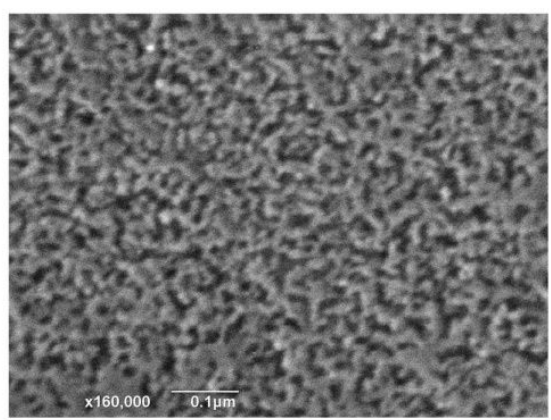

b

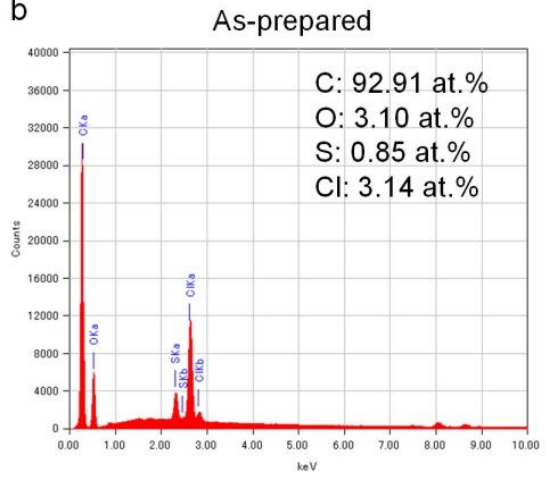

C Annealed at $900^{\circ} \mathrm{C}$ in $\mathrm{Ar}$

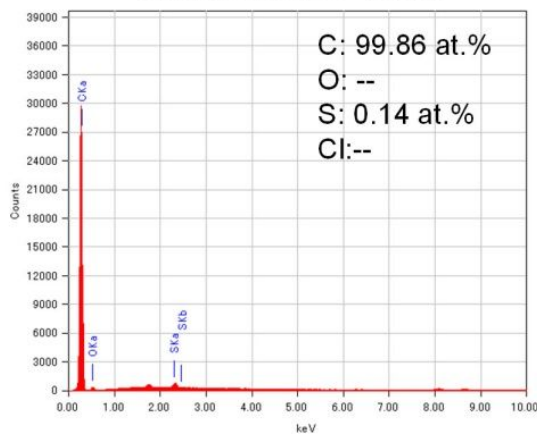

Figure S2. (a) Low-magnification SEM image of the a-C. (b,c) Compositional analyses by SEM-EDS of a-C before and after thermal annealing at $900^{\circ} \mathrm{C}$ in $\mathrm{Ar}$. 

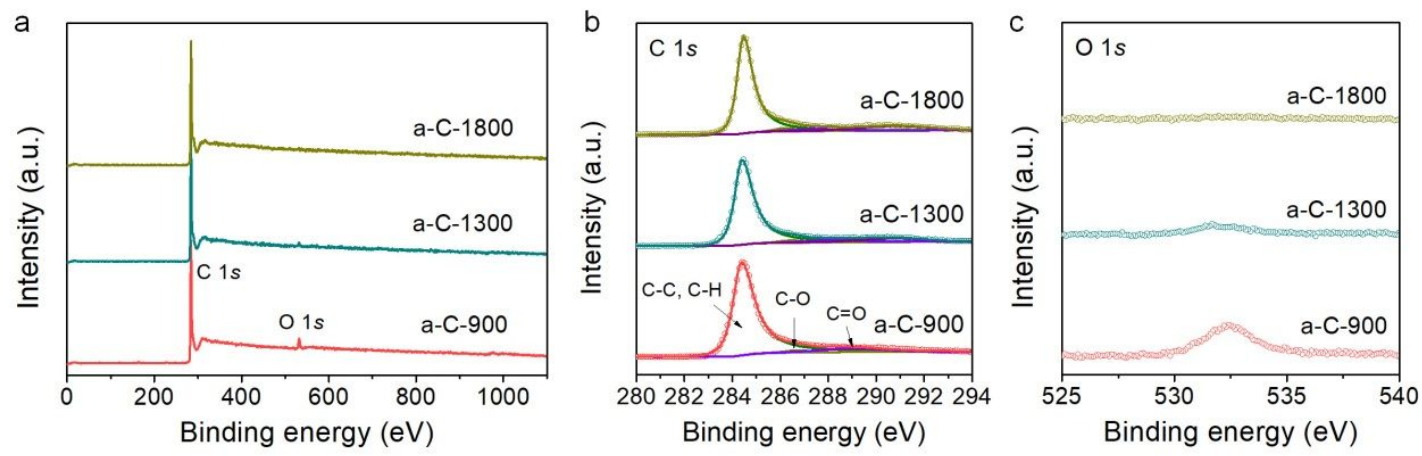

d

\begin{tabular}{cccccc}
\hline & $\begin{array}{c}\mathrm{C} \mathrm{1s} \\
\text { [at.\%] }\end{array}$ & $\begin{array}{c}\text { O 1s } \\
\text { [at.\%] }\end{array}$ & $\begin{array}{c}\mathrm{Cl} \mathrm{2p} \\
\text { [at.\%] }\end{array}$ & $\begin{array}{c}\mathrm{Ni} 2 \mathrm{p} \\
\text { [at.\%] }\end{array}$ & $\begin{array}{c}\mathrm{Fe} \mathrm{2p} \\
\text { [at.\%] }\end{array}$ \\
\hline a-C-900 & 96.8 & 3.2 & none & none & none \\
a-C-1300 & 98.6 & 1.4 & none & none & none \\
a-C-1800 & 99.6 & 0.4 & none & none & none \\
\hline
\end{tabular}

Figure S3. (a) XPS survey spectra for a-C-900, a-C-1300 and a-C-1800. (b-c) The fine-scanned XPS spectra for $\mathrm{C} 1 s$ and O $1 s$. (d) Elemental analysis for the a-C samples. The abundant adsorbates of $\mathrm{O}$ - and $\mathrm{Cl}$-containing functional groups in as-prepared a-C have been removed after the thermal treatments. 

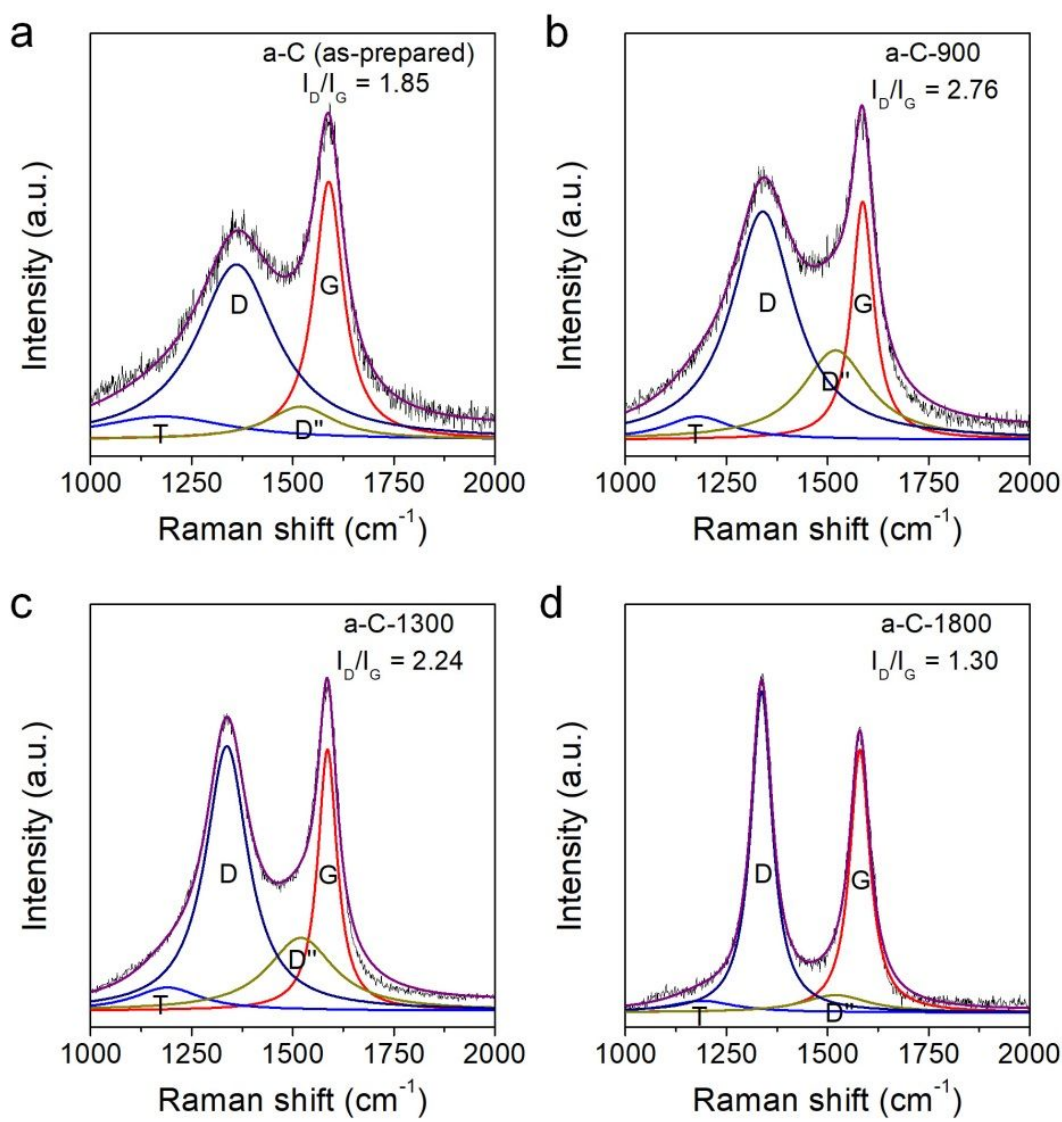

Figure S4. Fitted Raman spectra of (a) a-C (as-prepared), (b) a-C-900, (c) a-C-1300, and (d) a-C-1800. 

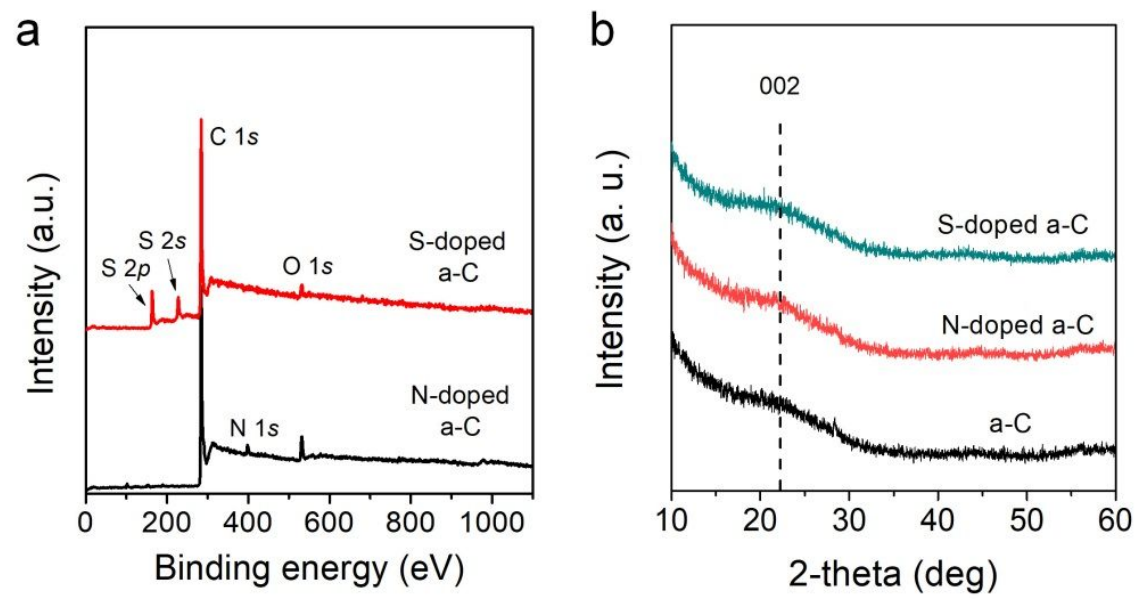

Figure S5. XPS and XRD characterizations of the N- and S-doped a-C prepared by annealing the as-prepared a-C samples in an ammonia or sulfur vapor atmosphere at $700^{\circ} \mathrm{C}$. (a) XPS survey spectra for $\mathrm{N}$ - and $\mathrm{S}$-doped a-C, showing distinct nitrogen and sulfur signals. The $\mathrm{N}$-doped a-C contains 2.6 at.\% N, the S-doped a-C contains 6.9 at.\% S. (b) XRD patterns showing very broad and weak diffraction peaks for all samples. Particularly, while conventional N- and S-doped amorphous carbons typically show dopant-induced graphitic interlayer expansions $\mathrm{s}^{2-4}$, no shift of the broad XRD (002) peak occurs for both the N- and S-doped a-C. This result further supports the fact that a-C processed at this moderate temperature has a fully disordered carbon structure with almost no graphitic domains. 

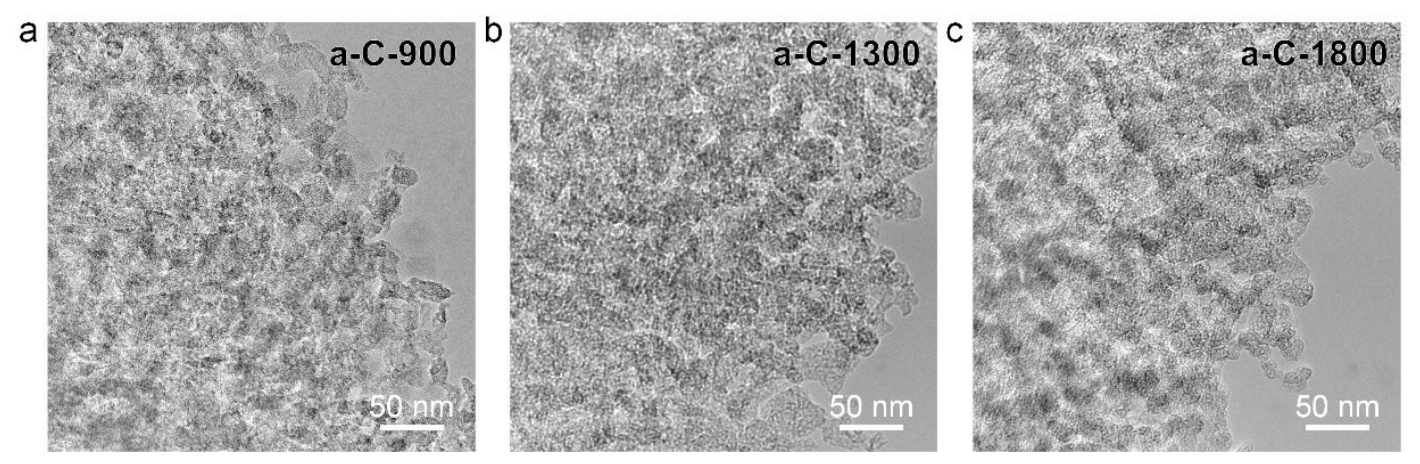

Figure S6. Low-magnification TEM images of a-C annealed at different temperatures in Ar. The primary mesoporous structure is well retained for a-C-900, a-C-1300 and a-C-1800. 

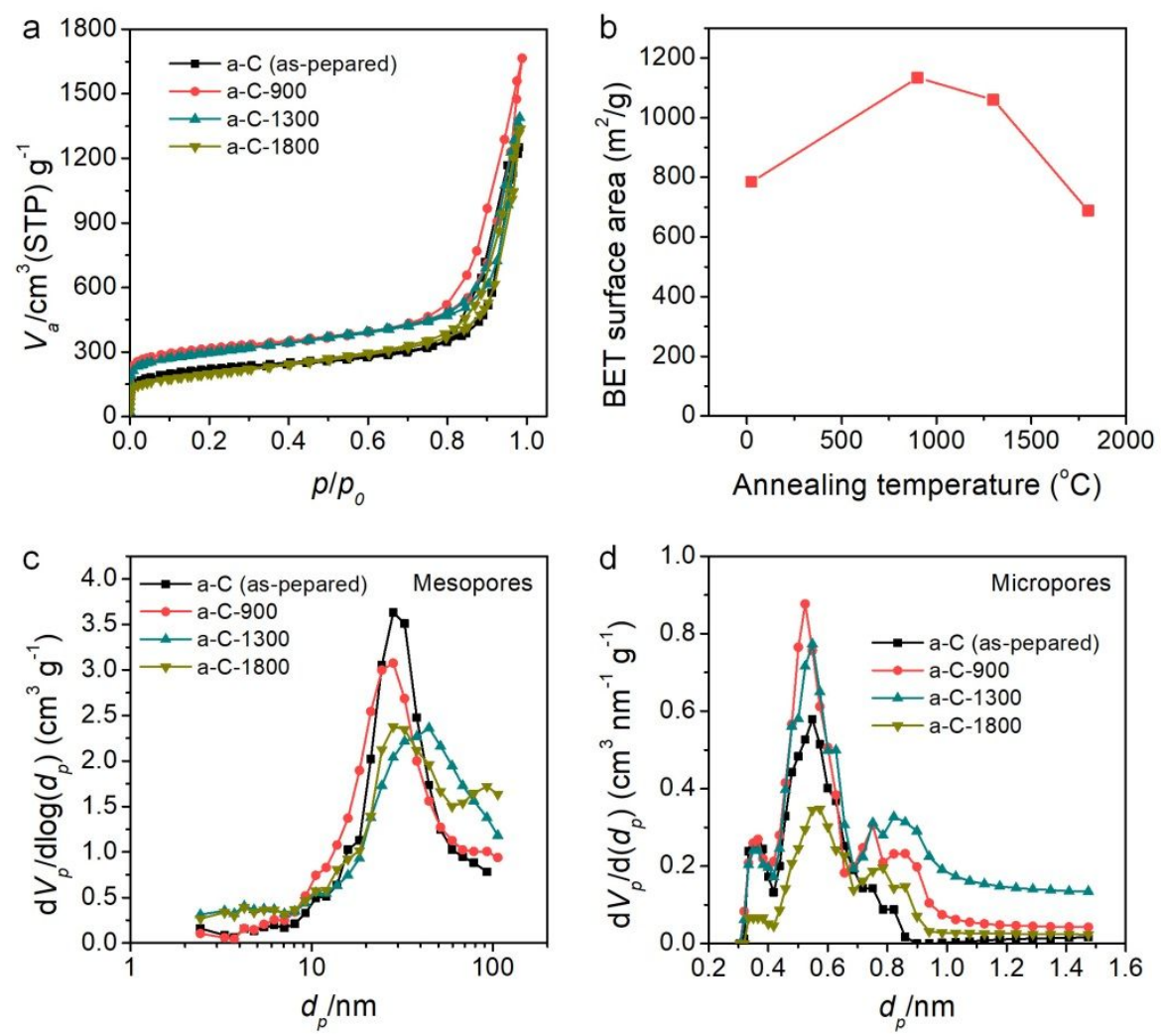

Figure S7. (a) Nitrogen adsorption/desorption isotherms for a-C annealed at different temperatures in Ar. (b) BET surface areas. (c) Pore size distributions determined by $\mathrm{N}_{2}$ gas sorption. (d) Pore size distributions determined by $\mathrm{CO}_{2}$ gas sorption. 

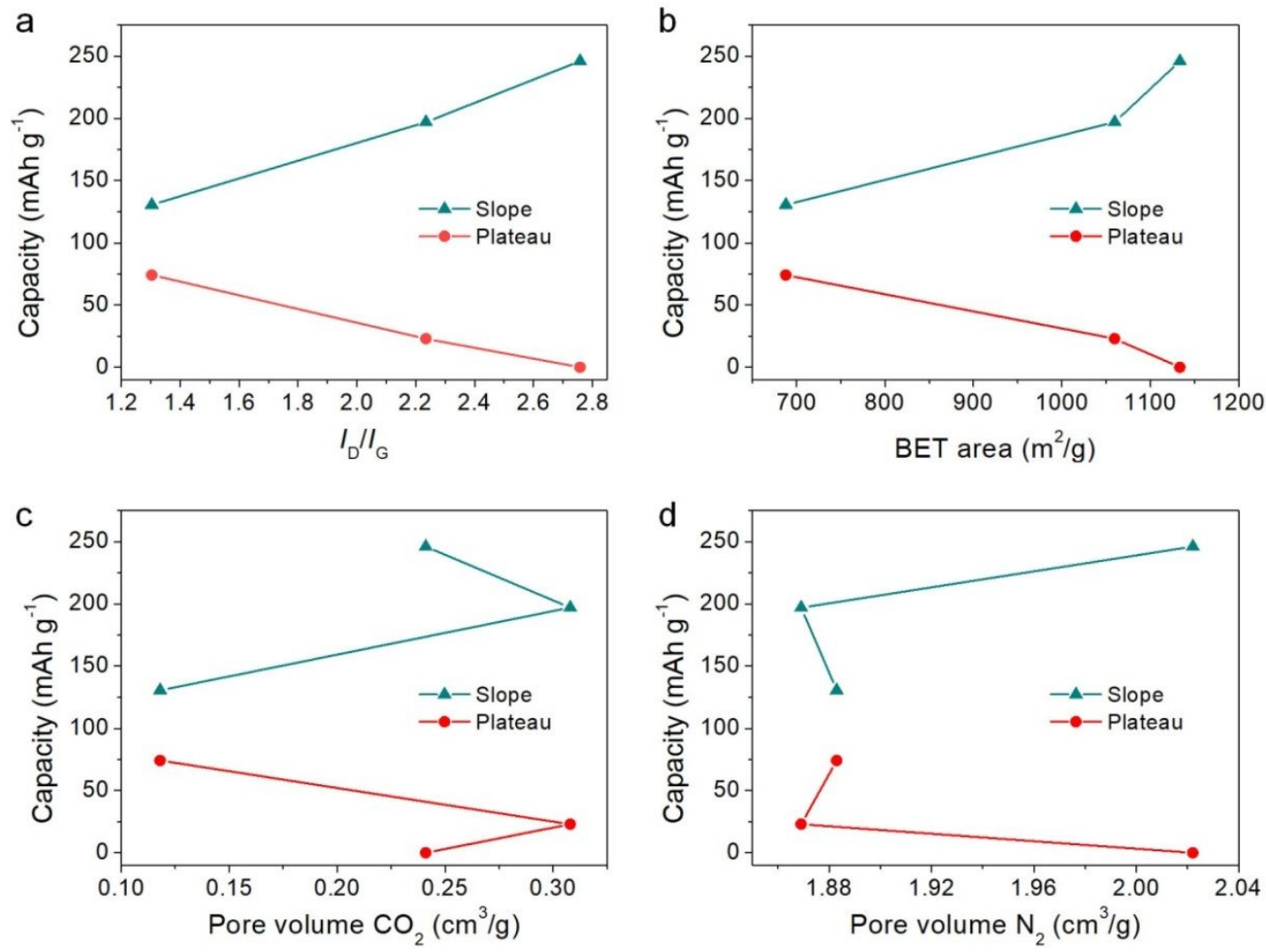

Figure S8. The sloping and plateau region capacity as a function of (a) $I_{\mathrm{D}} / I_{\mathrm{G}}$, (b) BET surface area, (c) micropore volume determined by $\mathrm{CO}_{2}$ gas adsorption measurement, and (d) total volume determined by $\mathrm{N}_{2}$ gas adsorption measurement. 
a

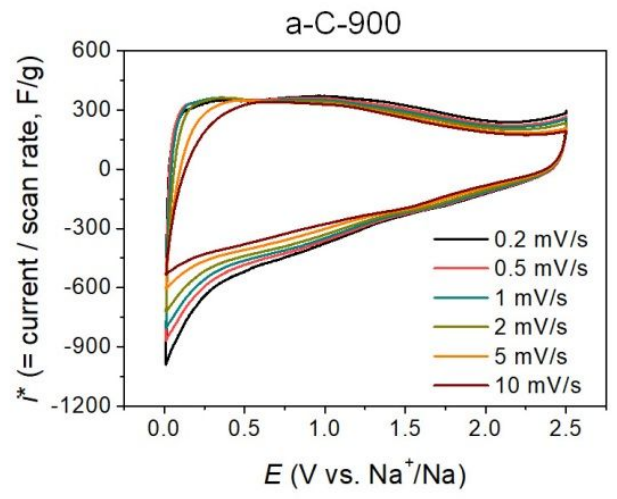

b
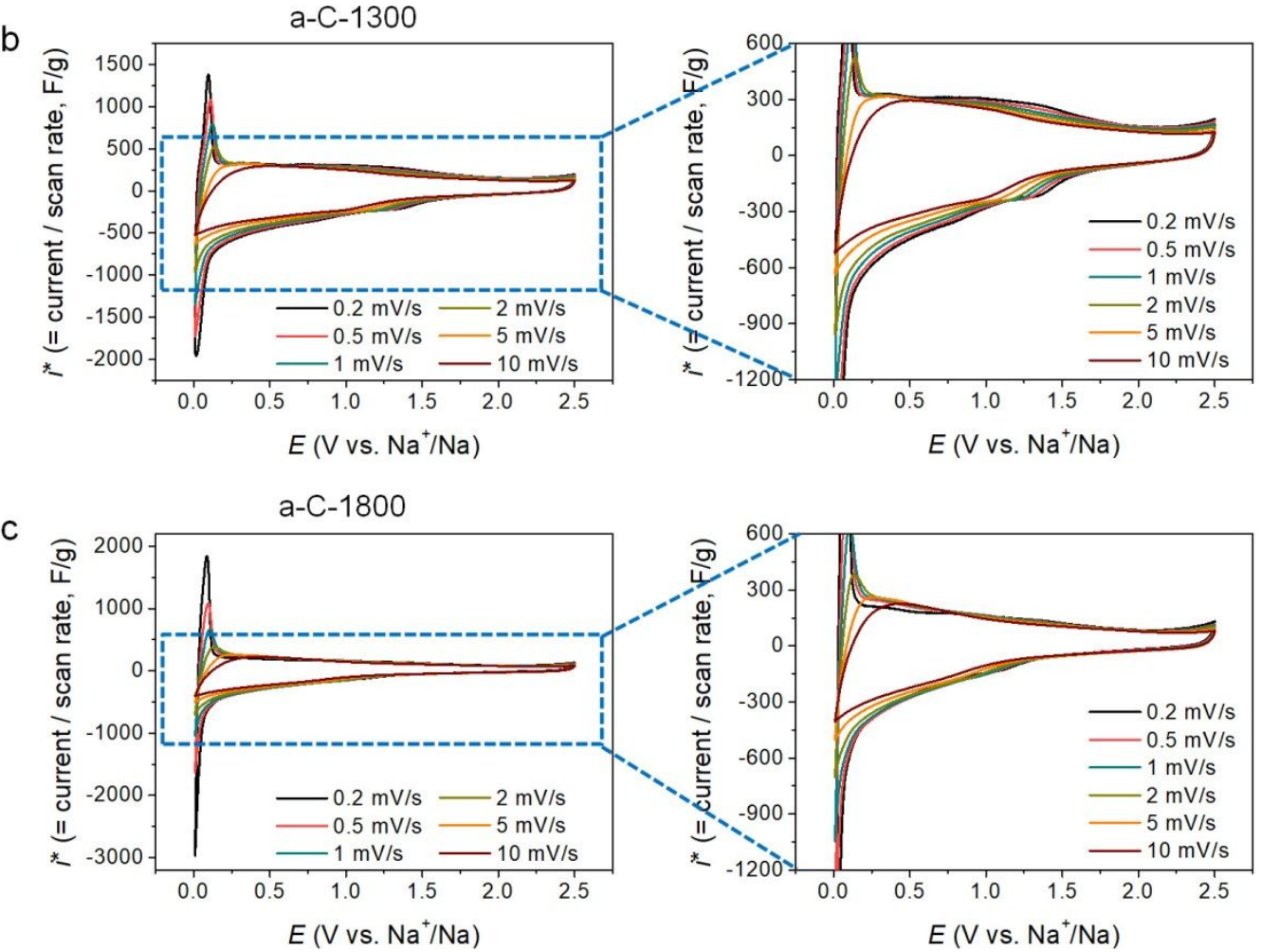

Figure S9. CV curves collected at various sweep rates for a-C-900, a-C-1300 and a-C-1800. The normalized current $i^{*}=$ current $(i) /$ sweep rate $(v)$. 
a-C-1300
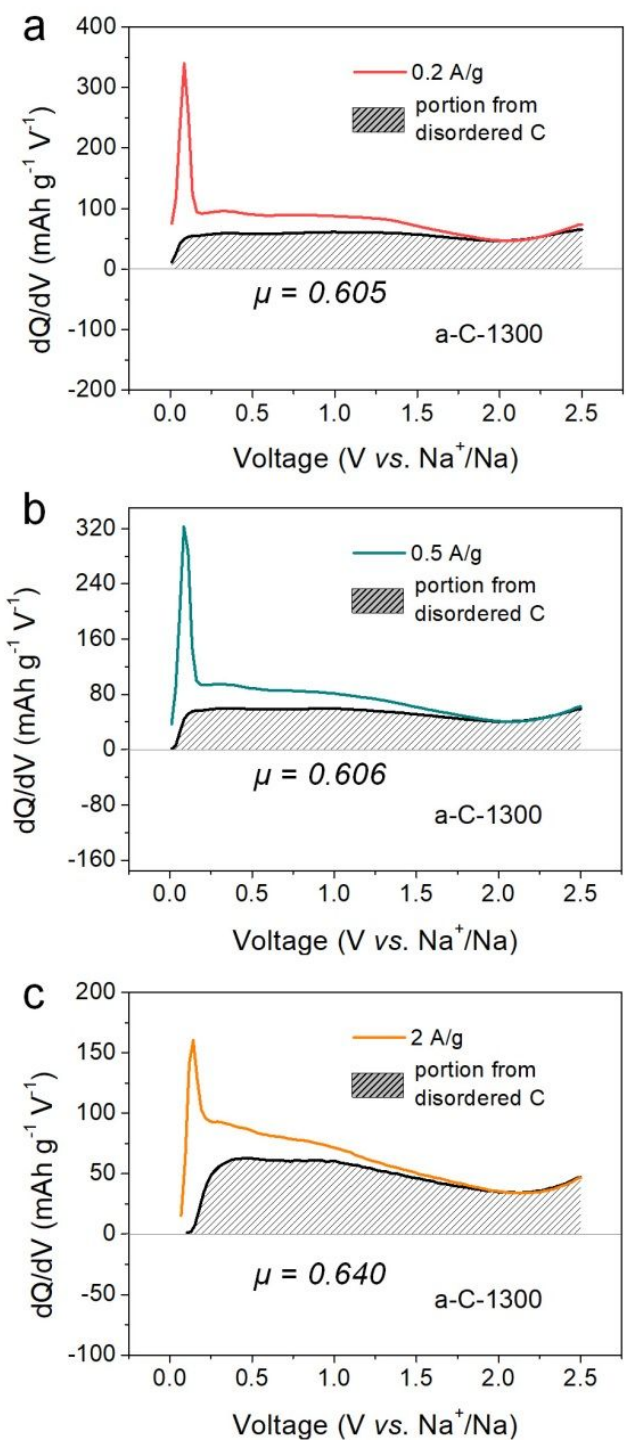

a-C-1800
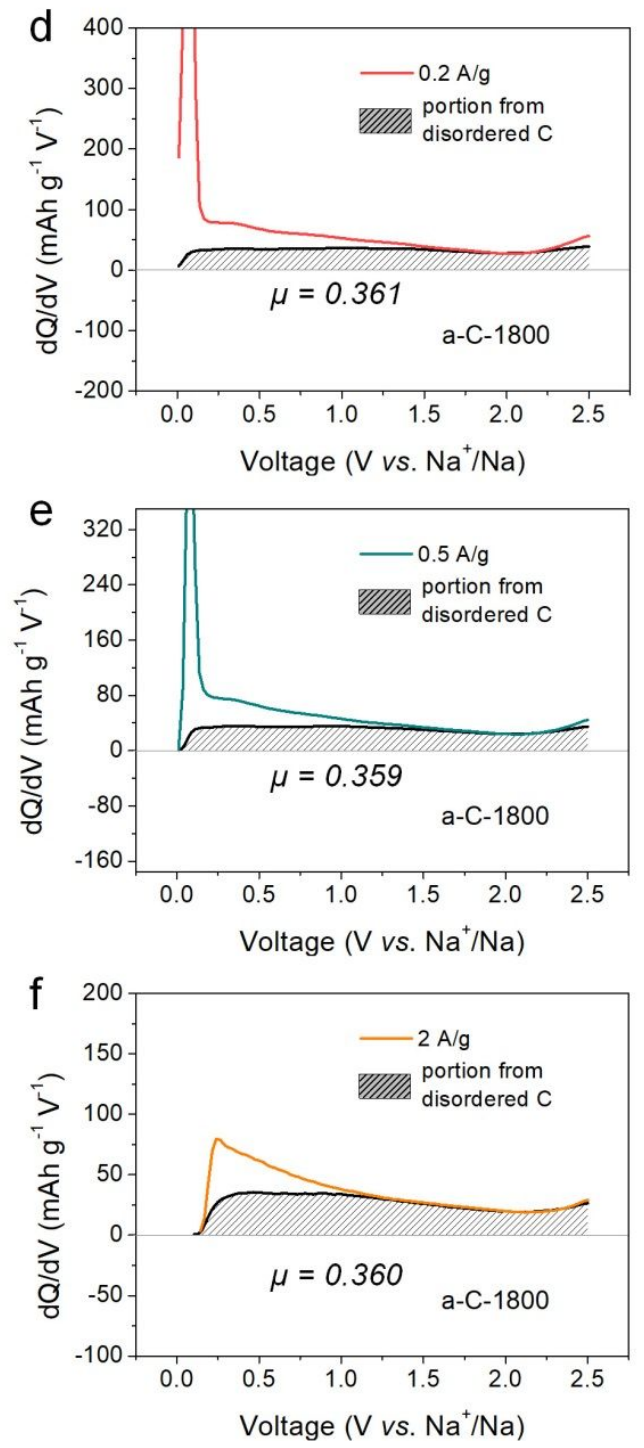

Figure S10. dQ/dV curves of the charging process at various current densities for a-C-1300 and a-C-1800. The hatched portions show charge contributions from the fully disordered carbon component. The $\mu$ values are given. 


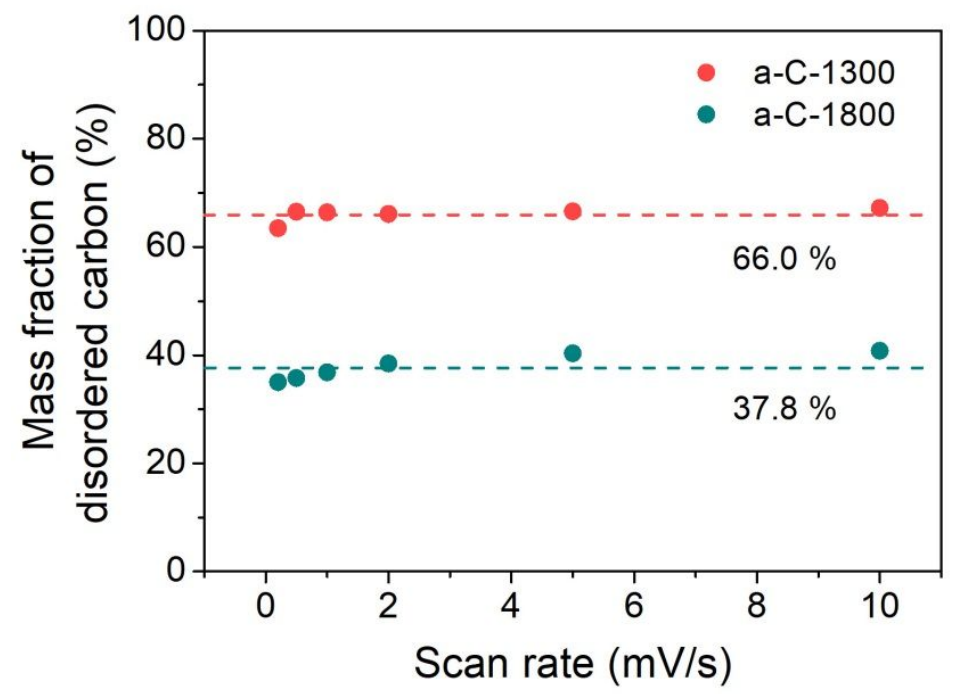

Figure S11. Mass fractions of the disordered carbon component for a-C-1300 and a-C-1800 obtained by analyzing the CV curves.
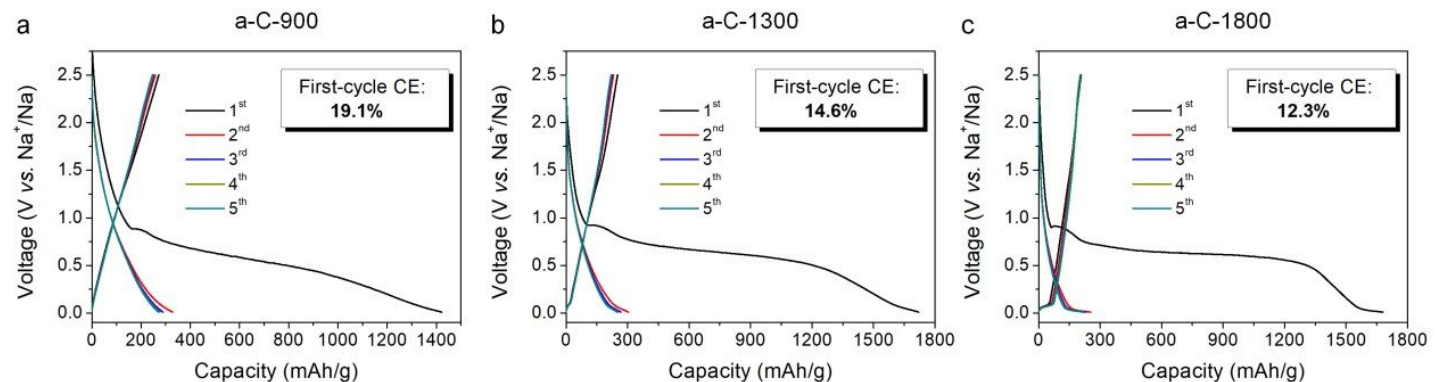

Figure S12. Galvanostatic charge/discharge profiles at $0.1 \mathrm{~A} / \mathrm{g}$ of the first five cycles for a-C-900, a-C-1300 and a-C-1800. The long plateaus at $\sim 0.6 \mathrm{~V}$ of the first discharge are associated with electrolyte decomposition to form the solid electrolyte interphase (SEI) films. It has been reported that the first-cycle Coulombic efficiency of a-C-900 can be greatly improved to $\sim 73.8 \%$ by a pre-sodiation surface treatment. ${ }^{1}$ 
Table S1. Physical parameters for the a-C materials

\begin{tabular}{|c|c|c|c|c|c|c|c|c|c|c|}
\hline Sample & $\begin{array}{c}(002) \\
\text { Peak } \\
\text { position } \\
\end{array}$ & $\begin{array}{c}d_{002} \\
(\mathrm{~nm})\end{array}$ & $\begin{array}{c}L_{\mathrm{c}} \\
(\mathrm{nm})\end{array}$ & $\boldsymbol{I}_{\mathrm{D}} / \boldsymbol{I}_{\mathrm{G}}$ & $\begin{array}{c}L_{\mathrm{a}} \\
(\mathbf{n m})\end{array}$ & $\begin{array}{c}S_{\mathrm{BET}} \\
\left(\mathrm{m}^{2} / \mathrm{g}\right)\end{array}$ & $\begin{array}{c}\text { Mesopore } \\
\text { size }(n m)\end{array}$ & $\begin{array}{c}\text { Micropore } \\
\text { size }(\mathbf{n m})\end{array}$ & $\begin{array}{c}\text { Total pore } \\
\text { volume } \\
\left(\mathrm{cm}^{3} / \mathrm{g}\right)\end{array}$ & $\begin{array}{c}\text { Micropore } \\
\text { volume } \\
\left(\mathrm{cm}^{3} / \mathrm{g}\right) \\
\end{array}$ \\
\hline $\begin{array}{c}\text { a-C } \\
\text { (as-prepared) }\end{array}$ & -- & -- & -- & 1.85 & -- & 785 & 28.2 & 0.55 & 1.73 & 0.15 \\
\hline a-C-900 & 22.33 & 0.398 & 0.96 & 2.76 & 6.10 & 1134 & 24.4 & 0.52 & 2.02 & 0.24 \\
\hline a-C-1300 & 22.74 & 0.391 & 1.07 & 2.24 & 7.52 & 1060 & 28.2 & 0.55 & 1.87 & 0.31 \\
\hline $\mathrm{a}-\mathrm{C}-1800$ & 23.48 & 0.379 & 1.11 & 1.30 & 12.90 & 688 & 28.2 & 0.57 & 1.88 & 0.12 \\
\hline
\end{tabular}


Table S2. Comparison of $L_{\mathrm{a}}$ and $L_{\mathrm{c}}$ between the a-C and other hard and soft carbon materials

\begin{tabular}{|c|c|c|c|c|c|}
\hline Sample & $d_{002}(\mathrm{~nm})$ & $L_{\mathrm{c}}(\mathrm{nm})$ & $I_{\mathrm{D}} / I_{\mathrm{G}}$ & $L_{\mathrm{a}}(\mathrm{nm})$ & Ref. \\
\hline a-C-900 & 0.398 & 0.96 & 2.76 & 6.10 & This work \\
\hline $\mathrm{a}-\mathrm{C}-1300$ & 0.391 & 1.07 & 2.24 & 7.52 & This work \\
\hline a-C-1800 & 0.379 & 1.11 & 1.30 & 12.90 & This work \\
\hline $\begin{array}{l}\text { Pitch-derived soft carbon } \\
\text { PC600 }\end{array}$ & 0.354 & 1.74 & 2.15 & 8.93 & \multirow{6}{*}{$\begin{array}{c}\text { Angew. Chem. Int. } \\
\text { Ed. } \\
\text { 2019, 58, } 4361 .\end{array}$} \\
\hline $\begin{array}{l}\text { Pitch-derived soft carbon } \\
\text { PC700 }\end{array}$ & 0.358 & 1.50 & 2.24 & 8.58 & \\
\hline $\begin{array}{l}\text { Pitch-derived soft carbon } \\
\text { PC } 800\end{array}$ & 0.360 & 1.47 & 2.57 & 7.47 & \\
\hline $\begin{array}{l}\text { Pitch-derived soft carbon } \\
\text { PC900 }\end{array}$ & 0.360 & 1.58 & 2.38 & 8.08 & \\
\hline $\begin{array}{l}\text { Pitch-derived soft carbon } \\
\text { PC1000 }\end{array}$ & 0.359 & 1.60 & 1.99 & 9.68 & \\
\hline $\begin{array}{l}\text { Pitch-derived soft carbon } \\
\text { PC1200 }\end{array}$ & 0.358 & 2.35 & 1.67 & 11.50 & \\
\hline $\begin{array}{l}\text { PTCDA-derived soft carbon } \\
\text { C-700 }\end{array}$ & 0.362 & 1.37 & -- & -- & \multirow{4}{*}{$\begin{array}{l}\text { ACS Cent. Sci. } \\
2015,1,516 .\end{array}$} \\
\hline $\begin{array}{l}\text { PTCDA-derived soft carbon } \\
\text { C-900 }\end{array}$ & 0.356 & 1.72 & -- & -- & \\
\hline $\begin{array}{l}\text { PTCDA-derived soft carbon } \\
\text { C-1100 }\end{array}$ & 0.353 & 2.19 & -- & -- & \\
\hline $\begin{array}{l}\text { PTCDA-derived soft carbon } \\
\text { C-1600 }\end{array}$ & 0.346 & 4.79 & -- & -- & \\
\hline $\begin{array}{l}\text { Sucrose-derived hard carbon } \\
\text { HC-5 }\end{array}$ & 0.413 & 1.30 & 3.23 & 5.96 & \multirow{4}{*}{$\begin{array}{l}\text { Adv. Energy Mater. } \\
2018,8,1703238 .\end{array}$} \\
\hline $\begin{array}{l}\text { Sucrose-derived hard carbon } \\
\text { HC- } 2\end{array}$ & 0.401 & 1.35 & 2.50 & 7.69 & \\
\hline $\begin{array}{l}\text { Sucrose-derived hard carbon } \\
\text { HC-1 }\end{array}$ & 0.398 & 1.42 & 2.38 & 8.07 & \\
\hline $\begin{array}{l}\text { Sucrose-derived hard carbon } \\
\text { HC- } 0.5\end{array}$ & 0.391 & 1.46 & 1.92 & 10.00 & \\
\hline $\begin{array}{l}\text { Phenolic resin-derived hard } \\
\text { carbon } \mathrm{HC} 1100^{\circ} \mathrm{C}\end{array}$ & 0.396 & 1.0 & 1.43 & 13.44 & \multirow{2}{*}{$\begin{array}{c}\text { Carbon } \\
2018,139,248 .\end{array}$} \\
\hline $\begin{array}{l}\text { Phenolic resin-derived hard } \\
\text { carbon } \mathrm{HC} 1300^{\circ} \mathrm{C}\end{array}$ & 0.387 & 1.0 & 1.29 & 14.90 & \\
\hline $\begin{array}{l}\text { Filter paper-derived hard } \\
\text { carbon }\end{array}$ & 0.383 & -- & 1.26 & 13.30 & $\begin{array}{l}\text { Chem. Commun. } \\
\text { 2017, 53, } 2610 .\end{array}$ \\
\hline $\begin{array}{l}\text { Argan shell-derived hard } \\
\text { carbon } 800 \mathrm{~W}\end{array}$ & 0.391 & 1.01 & 1.77 & 10.86 & \multirow{4}{*}{$\begin{array}{l}\text { J. Mater. Chem. A } \\
\text { 2017, 5, } 9917 .\end{array}$} \\
\hline $\begin{array}{l}\text { Argan shell-derived hard } \\
\text { carbon } 1000 \mathrm{~W}\end{array}$ & 0.39 & 0.96 & 1.78 & 10.80 & \\
\hline $\begin{array}{l}\text { Argan shell-derived hard } \\
\text { carbon } 1200 \mathrm{~W}\end{array}$ & 0.393 & 1.14 & 1.77 & 10.86 & \\
\hline $\begin{array}{l}\text { Argan shell-derived hard } \\
\text { carbon } 1300 \mathrm{~W}\end{array}$ & 0.385 & 1.09 & 1.76 & 10.92 & \\
\hline $\begin{array}{c}\text { Sucrose--derived hard carbon } \\
\text { HC }\end{array}$ & 0.377 & -- & 1.30 & 12.90 & \multirow{2}{*}{$\begin{array}{l}\text { Adv. Energy Mater. } \\
\text { 2017, 7, } 1602894 .\end{array}$} \\
\hline $\begin{array}{c}\text { Sucrose--derived hard carbon } \\
\text { P-HC }\end{array}$ & 0.395 & -- & 1.39 & 12.40 & \\
\hline
\end{tabular}




\begin{tabular}{cccccc}
$\begin{array}{c}\text { Sucrose--derived hard carbon } \\
\text { S-HC }\end{array}$ & 0.383 & -- & 1.18 & 14.20 & \\
$\begin{array}{c}\text { Sucrose--derived hard carbon } \\
\text { B-HC }\end{array}$ & 0.378 & -- & 1.15 & 14.60 & \\
$\begin{array}{c}\text { Cellulose-derived hard carbon } \\
\text { HC-900 }\end{array}$ & 0.388 & 1.45 & 1.85 & 10.38 & \\
$\begin{array}{c}\text { Cellulose-derived hard carbon } \\
\text { HC-1100 }\end{array}$ & 0.383 & 1.76 & 1.61 & 11.92 & Adv. Energy Mater. \\
$\begin{array}{c}\text { Cellulose-derived hard carbon } \\
\text { HC-1300 }\end{array}$ & 0.377 & 1.83 & 1.49 & 12.88 & 2017, 1700403. \\
$\begin{array}{c}\text { Cellulose-derived hard carbon } \\
\text { HC-1500 }\end{array}$ & 0.374 & 2.01 & 1.37 & 14.03 & \\
\hline
\end{tabular}

\section{References}

1. Han J. H.; Li H. P.; Lu Z.; Huang G.; Johnson I.; Watanabe K.; Chen M. W. 3D bimodal porous amorphous carbon with self-similar porosity by low-temperature sequential chemical dealloying. Chem. Mater. 2021, 3, 1013-1021.

2. Hong Z.; Zhen Y.; Ruan Y.; Kang M.; Zhou K.; Zhang J. M.; et al. Rational design and general synthesis of S-doped hard carbon with tunable doping sites toward excellent Na-ion storage performance. Adv. Mater. 2018, 30, 1802035.

3. Yang J.; Zhou X.; Wu D.; Zhao X.; Zhou Z. S-doped N-rich carbon nanosheets with expanded interlayer distance as anode materials for sodium-ion batteries. Adv. Mater. 2017, $29,1604108$.

4. Li Z.; Bommier C.; Chong Z. S.; Jian Z.; Surta T. W.; Wang X.; et al. Mechanism of Na-ion storage in hard carbon anodes revealed by heteroatom doping. Adv. Energy Mater. 2017, 7, 1602894. 